PRAMANA

- journal of

physics (c) Indian Academy of Sciences

pp. $1-20$

\title{
QGP Theory: Status and Perspectives
}

\author{
Steffen A. Bass \\ Department of Physics, Duke University \\ \& RIKEN-BNL Research Center, Brookhaven National Laboratory
}

\begin{abstract}
The current status of Quark-Gluon-Plasma Theory is reviewed. Special emphasis is placed on QGP signatures, the interpretation of current data and what to expect from RHIC in the near future.
\end{abstract}

Keywords. Quark-Gluon-Plasma, Heavy-Ion Collisions

\section{Introduction}

Ultra-relativistic heavy ion collisions offer the unique opportunity to probe highly excited dense nuclear matter under controlled laboratory conditions. One of the main driving forces for these studies is the expectation that an entirely new form of matter may be created in such reactions. This form of matter, called the Quark Gluon Plasma (QGP), is the QCD analogue of the plasma phase of ordinary atomic matter. However, unlike such ordinary plasmas, the deconfined quanta of a QGP are not directly observable because of the fundamental confining property of the physical QCD vacuum. What is observable are hadronic and leptonic residues of the transient QGP state. The QGP state formed in nuclear collisions is a transient rearrangement of the correlations among quarks and gluons contained in the incident baryons into a larger but globally still color neutral system with however remarkable theoretical properties, such as restored chiral symmetry and deconfinement. The task with heavy ion reactions is to provide experimental information on this fundamental prediction of the Standard Model (for recent reviews on QGP signatures, please see [1]).

\section{The QCD Phase Diagram}

QCD is a non-abelian gauge theory, it's basic constituents are quarks and anti-quarks interacting through the exchange of color-charged gluons. At very high temperatures and densities, in the domain of weak coupling between quarks and gluons, long range interactions are dynamically screened [2,3]. Quarks and gluons are then no longer confined to bound hadronic states ("deconfinement"). Furthermore, chiral symmetry is restored - for baryon-free matter - apparently at the same temperature $T_{C}$.

Lattice gauge simulations of QCD [4,5], provide the only rigorous method to compute the equation of state of strongly interacting elementary particle matter. In principle both, 
3-flavour phase diagram

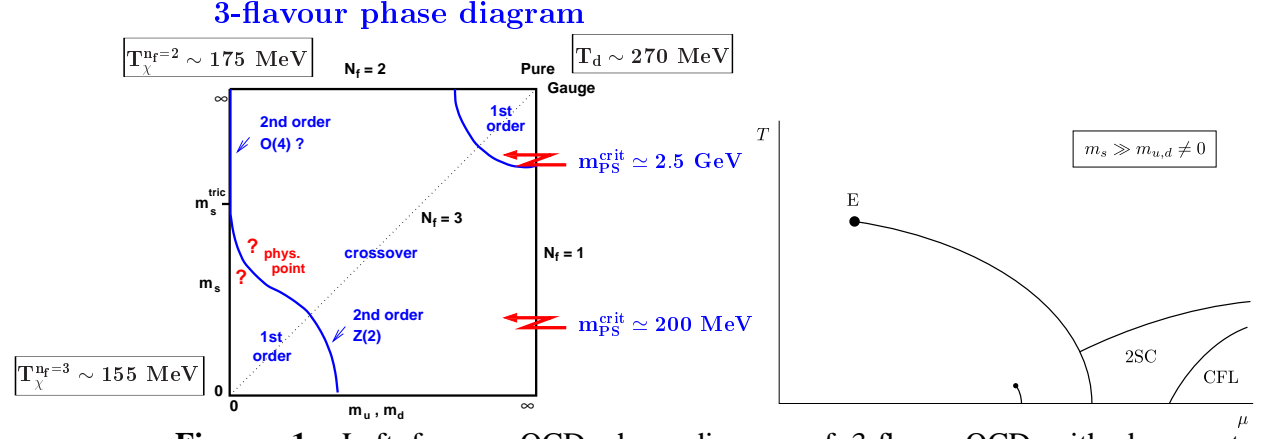

Figure 1. Left frame: QCD phase diagram of 3-flavor QCD with degenerate (u,d)-quark masses and a strange quark mass $m_{s}$. Right frame: schematic QCD phase diagram as a function of $\mu_{B}$.

the non-perturbative hadronic matter and the non-perturbative QGP phases of QCD can be investigated. The main disadvantage of lattice simulations is the current practical restriction to finite, periodic, baryon free systems in global equilibrium, a scenario far away from the highly inhomogeneous off-equilibrium situation found in complex heavy-ion reactions.

Lattice calculations yield a critical temperature of $T_{C} \approx 270 \mathrm{MeV}$ in the quenched approximation [6,7] - where neither dynamical quarks, nor a chiral phase transition exist. Simulations including dynamical quarks at $\mu_{B}=0$ indicate a critical temperature in the order of $T_{C} \approx 165 \mathrm{MeV}$ (see figure 1, taken from [7]).

Many striking QGP signatures depend heavily on the assumption of a first order phase transition and the existence of a mixed phase of QCD matter. However, both, the order of the phase transition as well as the critical temperature, depend on the parameters of the calculation, namely the number of quark flavors and their masses. For the most realistic case of QCD with two flavors of light quarks with masses between 5 and $10 \mathrm{MeV}$ and one flavor with a mass around $200 \mathrm{MeV}$, the situation remains unclear: the order of the phase transition seems to depend on the numerical values for the masses of the light and heavy quarks [8,7]. If the latter is too heavy, the transition might be smeared out to a mere rapid increase of the energy density over a small temperature interval. In this case the use of simple deconfinement scenarios may lead to wrong expectations for observables. The elementary excitations in such a phase transition region ought not be described by quarks and gluons but could physically resemble more hadronic excitations with strongly modified "in-medium" properties [9].

This raises a practical question, whether conclusions based on $\mu_{B}=0$ estimates, might misguide physical argumentation for observables in nuclear collisions. This warning is particularly appropriate for those QGP-signals, where a 50\% quantitative change of an observable is used to differentiate QGP production scenarios from ordinary hadronic transport ones.

The inclusion of the second most important thermodynamic variable, the chemical potential $\mu_{B}$ into a full fletched lattice-QCD calculation has recently been attempted [10], but still carries large systematical uncertainties. These calculations predict the existence of a tricritical point - only for $\mu_{B}>\mu_{B, \text { crit }}$ would a phase-boundary with a possibly 1st order phase-transition exist.

An additional complication is that for systems of finite volume $\left(\mathrm{V} \leq 125 \mathrm{fm}^{3}\right)$ the de- 
QGP Theory: Status and Perspectives

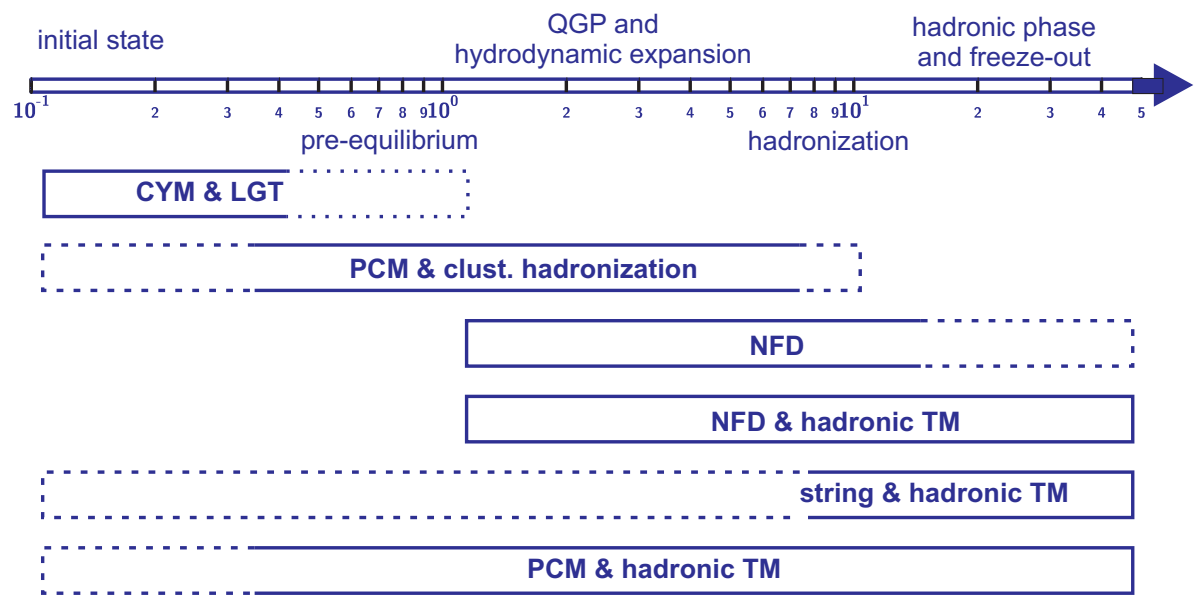

Figure 2. Transport theory approaches for RHIC and their range of applicability.

confinement cannot be complete. Fluctuations lead to a finite probability of the hadronic phase above $T_{C}$. The sharp discontinuity (e.g. $\varepsilon / T^{4}$ ) is thus smeared out [11].

Recently, a lot of progress has been made towards understanding the QCD phase diagram at large chemical potential $\mu_{B}$ [12]. At very high densities, asymptotic freedom and BCS theory have been utilized to predict new, superconducting, phases of QCD matter. Depending on the mass of the strange quark $\left(m_{s} \gg m_{u, d}\right)$, first a two flavor colorsuperconductor $\left(m_{s} \rightarrow \infty\right)$ or a color-flavor locked superconducting phase $\left(m_{s}\right.$ decreasing with $\mu_{B}$ ) are found (see right frame of figure 1, taken from [13]). The color-flavor locking refers to the symmetry of the ground state only being given under a simultaneous transformation of color and flavor. It should be noted, however, that these new phases of high-density QCD will most likely not be found in heavy-ion reactions (the temperature in such collisions is too high for color superconductivity to occur), but may rather have a large impact in our understanding of matter in neutron stars and other cold super-dense objects (for a review on the topic, please see [13]).

\section{Dynamics: Transport Theory at SPS and RHIC}

Transport Theory offers the unique capability of connecting experimentally observable quantities in a relativistic heavy ion collision with its time evolution and reaction dynamics, thus giving crucial insights into the possible formation of a transient deconfined phase of hot and dense matter, the Quark-Gluon-Plasma.

Figure 2 provides an overview of different transport theoretical ansatzes currently in the literature for the description of a relativistic heavy ion collision at RHIC energies. The timeline shows a best case scenario for what to expect: the formation of a thermalized QGP with subsequent hadronization and freeze-out. Bands with solid lines denote the safe range of applicability for the respective transport approach, whereas dashed/dotted bands refer to areas in which the approach is still applied but where the assumptions on which the approach is based upon may be questionable or not valid anymore. 
The initial state and early pre-equilibrium phase are best described in Classical YangMills theory (CYM) [14] or Lattice Gauge Transport (LGT) [15] calculations - only these classes of models treat the coherence of the initial state correctly, but do not provide any meaningful dynamics for the later reaction stages.

The Parton Cascade Model (PCM) [16] and related pQCD approaches [33] treat the initial state as incoherent parton configuration, but are very well suited for the pre-equilibrium phase and subsequent thermalization, leading to a $Q G P$ and hydrodynamic expansion. Microscopic degrees of freedom in this ansatz are quarks and gluons which are propagated according to a Boltzmann Equation with a collision term using leading order pQCD cross sections. Augmented with a cluster hadronization ansatz the PCM is applicable up to hadronization. The range of this model can be even further extended if it is combined with a hadronic cascade which treats the hadronic phase and freeze-out correctly [18].

Nuclear Fluid Dynamics (NFD, see e.g. [19,20]) is ideally suited for the QGP and hydrodynamic expansion reaction phase, but breaks down in the later, dilute, stages of the reaction when the mean free paths of the hadrons become large and flavor degrees of freedom are important. The reach of NFD can also be extended by combining it with a microscopic hadronic cascade model - this kind of hybrid approach (dubbed hydro plus micro) was pioneered in [21] and has been now also taken up by other groups [22]. It is to date the most successful approach for describing the soft physics at RHIC. The biggest advantage of NFD is that it directly incorporates an equation of state as input - one of it's largest disadvantages is that it requires thermalized initial conditions and one is not able to do an ab-initio calculation.

Last but not least, string and hadronic transport models [23,24] have been very successful in the AGS and SPS energy domains. They treat the early reaction phase as a superposition of hadron-hadron strings and are thus ill suited to describe the microscopic reaction dynamics of deconfined degrees of freedom. In the later reaction stages, however, they are the best suited approach, since they incorporate the full spectrum of degrees of freedom of a hadron gas (including flavor dependent cross sections).

However, it is important to note that there is not a single transport theoretical ansatz currently available, which is able to cover the entire time-evolution of a collision at RHIC in one self-consistent approach.

\section{SPS: highlights and open questions}

During the last eight years the heavy ion research at the CERN-SPS has succeeded to achieve the measurement of a wide spectrum of observables relevant for a QGP search. Table 1 provides an overview of these measurements together with an assessment if the results would be compatible with the possible creation of a new form of deconfined matter $[25,26]$. In the following sections, several of these results will be highlighted in greater detail:

\subsection{Multi-strange Baryons}

The relative enhancement of strange and especially multistrange baryons with respect to peripheral (or proton induced) interactions has been suggested as a signature for the tran- 
QGP Theory: Status and Perspectives

\begin{tabular}{|l|l|c|}
\hline Concept & Observable & \\
\hline \hline creation of dense nuclear matter & stopping & $\sqrt{ }$ \\
\hline creation of high temperatures & energy spectra & $\sqrt{ }$ \\
\hline compression of nuclear matter & collective flow & $\sqrt{ }$ \\
\hline hadron source space-time evolution & particle interferometry & $\sqrt{ }$ \\
\hline remnants of hadronization & strangeness enhancement & $\sqrt{ }$ \\
\hline ashes of plasma & strangelets and hypermatter & $?$ \\
\hline restoration of chiral symmetry & masses/widths of vector mesons & $\sqrt{ }$ \\
\hline Debye-screening in a QGP & $J / \Psi$ and $\Psi^{\prime}$ suppression & $\sqrt{ }$ \\
\hline radiation of plasma & direct photons \& thermal dileptons & $\sqrt{ }$ \\
\hline
\end{tabular}

Table 1. Overview of concepts relevant for a QGP search and their corresponding observables investigated at the CERN/SPS. The last column indicates the success of the measurement and its compatibility with the possible creation of a new form of deconfined matter.

sient existence of a QGP-phase [27-29]: the main argument being that the (chemical or flavor) equilibration times should be much shorter in the plasma phase than in a thermally equilibrated hadronic fireball of $T \sim 160 \mathrm{MeV}$.

The dominant production mechanism in an equilibrated (gluon rich) plasma phase, namely the production of $s \bar{s}$ pairs via gluon fusion $(g g \rightarrow s \bar{s})$ [27], should allow for equilibration times similar to the interaction time of the colliding nuclei, and to the expected plasma lifetime (a few $\mathrm{fm} / \mathrm{c}$ ).

The yields of strange baryons per event calculated in a microscopic string/hadron transport model (UrQMD) are shown in the left frame of figure 3 as a function of the number of participants for $\mathrm{Pb}+\mathrm{Pb}$ and $\mathrm{p}+\mathrm{Pb}$ collisions at $160 \mathrm{GeV} / \mathrm{u}$ [30]. The $\Lambda+\bar{\Lambda}$ - (circles), $\Xi^{-}+\overline{\Xi^{-}}$- (squares), and $\Omega^{-}+\overline{\Omega^{-}}$- (triangles) values are shown. The stars correspond to experimental data of the WA97 collaboration [31]. Open symbols represent the results of the standard UrQMD calculations, whereas full symbols exhibit a calculation with an enhanced string tension of $\kappa=3 \mathrm{GeV} / \mathrm{fm}$, for the most central collisions $\left(N_{\text {part }} \geq 300\right)$. Obviously the standard UrQMD calculation, which can be seen as a baseline of known hadronic physics, strongly underestimates the (multi-)strange particle yields in central collisions, in particular in the case of the $\Omega^{-}$. Only the inclusion of non-hadronic medium effects, like color-ropes [32], which are simulated by increasing the string-tension for central collisions, enhances the yield to a level of near-compatibility with the data. Similar findings have also been made in the context of HIJING calculations [33]. While these findings by no means prove the validity of the color-rope approach, they clearly show the necessity of some kind of medium effect beyond regular binary hadronic (re)scattering in order to understand the data. Recently, hadronic multi-particle interactions in the early dense reaction phase have been suggested to significantly enhance the yield of anti-protons and (anti-)hyperons [34,35]. It remains to be seen, however, whether these effects are sufficient to explain the observed $\Omega^{-}$enhancement or other non-hadronic (i.e. deconfinement based) effects need to be taken into account. 

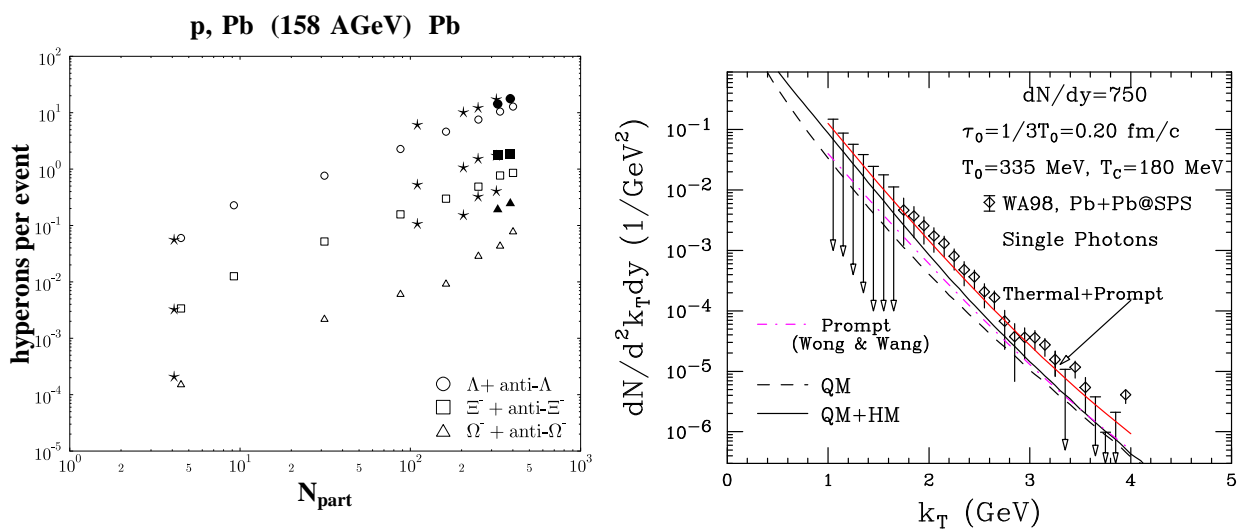

Figure 3. Left: excitation function of strange baryon multiplicity vs. number of participants in a microscopic transport approach. Right: Hydrodynamical analysis of direct photon production at the SPS.

\subsection{Penetrating Probes: Dileptons and Direct Photons}

Direct (thermal) photons in a QGP are created dominantly via $q \bar{q} \rightarrow \gamma g$ (annihilation) and $g q \rightarrow \gamma q$ (Compton scattering). The production rate and the momentum distribution of the photons depend on the momentum distributions of quarks, anti-quarks and gluons in the plasma. If the plasma is in thermodynamic equilibrium, the photons may carry information on this thermodynamic state at the moment of their production [36-39].

The main hadronic background processes to compete against are pion annihilation $\pi \pi \rightarrow$ $\gamma \rho$ and Compton scattering $\pi \rho \rightarrow \gamma \pi$ [36,40]. The broad $a_{1}$ resonance may act as an intermediate state in $\pi \rho$ scattering and thus provide an important contribution [41,40] via it's decay into $\gamma \pi$. In the vicinity of the critical temperature $T_{C}$ a hadron gas was shown to "shine" as brightly (or even brighter than) a QGP [36].

Hydrodynamical calculations can be used to compare purely hadronic scenarios of photon radiation with scenarios involving a first/second order phase transition to a QGP. They show a reduction in the temperature of the photon spectrum in the event of a first order phase-transition [42-44]. The right frame of figure 3 shows a hydrodynamical calculation assuming the creation of a QGP and subsequent hadronic rescattering [45]. Recent estimates of photon production in quark-matter (at two loop level) along with the dominant reactions in the hadronic matter leading to photons are used. About half of the radiated photons are seen to have a thermal origin. The same treatment and the initial conditions provide a very good description to hadronic spectra measured by several groups, lending additional support to the conclusion that quark gluon plasma has been formed in these collisions.

Dileptons carry information on the thermodynamic state of the medium at the moment of production in the very same manner as the direct photons - since the dileptons interact only electromagnetically they can leave the hot and dense reaction zone basically undistorted, too. It has been found that at the SPS the invariant mass spectrum of low-mass dileptons is dominated by pion-pion scattering. Medium modifications to the form-factor are necessary for a solid understanding of the data - the current status of experiment and theory point 

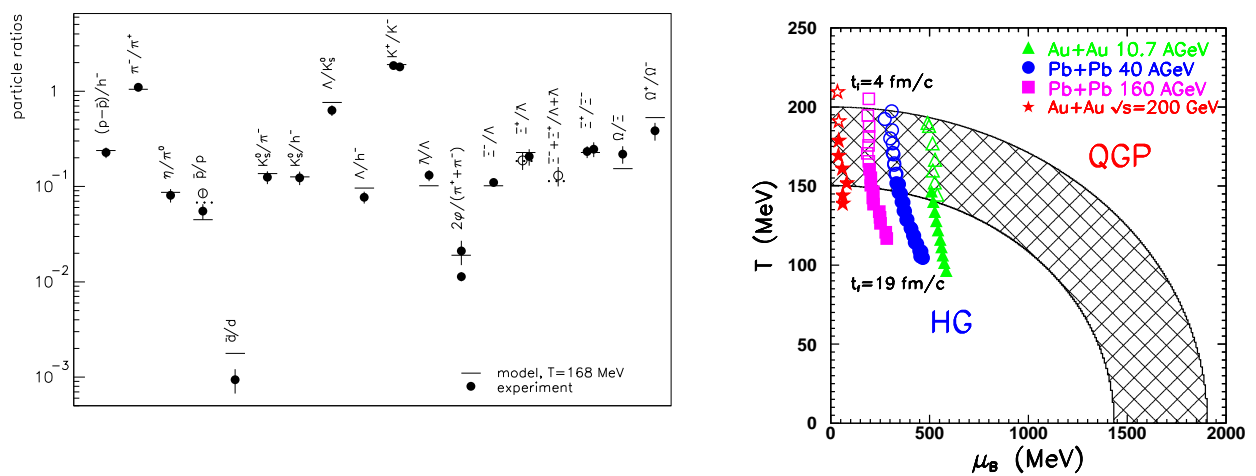

Figure 4. Left: statistical model fit to hadron ratios measured at the SPS. Right: statistical model analysis of the central cell of a heavy-ion collision calculated with a microscopic transport model.

toward a strong broadening of the $\rho$ resonance without a significant mass-shift toward lower masses [46].

\subsection{Chemical Equilibration}

The success of statistical models in describing the (strange) hadron abundances and ratios at the CERN/SPS [47] (see the left frame of figure 4, taken from [48]) and the extracted $\gamma_{s}$ values close to 1 have led to the common belief that chemical freeze-out in heavy-ion reactions at the SPS occurred very close to - or even at hadrochemical equilibrium and that this state most likely has been created by a hadronizing QGP $[25,26,49]$.

However, the estimated chemical equilibration times may not be sufficiently rapid to cause chemical equilibration before hadronization: calculations based on boost-invariant hydrodynamics with rate-equations for quark production [50-52], pQCD rate-equations [53] or the Parton Cascade Model [54] all indicate that chemical equilibration (and strangeness saturation) cannot be achieved during realistic life-times of the deconfined phase. It has been suggested (e.g. in [50,51]) that the system would be driven toward and come close to chemical equilibrium in the subsequent hadronic phase - a scenario which would help to bridge the gap between the calculations indicating insufficient equilibration time in the plasma phase and the SPS data apparently close to chemical equilibrium at chemical freeze-out. Recent calculations assuming a hadronizing QGP out of chemical equilibrium with subsequent hadronic rescatting have shown that rescatting via binary collisions in the hadronic phase is insufficient to drive the system toward chemical equilibrium before the expansion of the system leads to chemical freeze-out [55]. However, hadronic multi-particle collisions shortly after hadronization may have a large impact and drive the system more effectively toward chemical equilibrium [34,35] - a detailed calculation to verify/falsify this speculation has yet to be performed.

Another question which needs to be raised is whether the temperature and chemical potential extracted from the statistical model fits to final state hadron yields or ratios really 
reflect the thermodynamic state of the system at one particular time during its evolution (i.e. the conditions at chemical freeze-out) or are rather the result of a superposition of different states, due to individual hadron species decoupling continuously from the system (as would be expected from their different mean free paths). The latter view is supported by a transport model analysis of the time-evolution of the temperature and chemical potential in the central cell of a heavy-ion reaction (see the right frame of figure 4, taken from [56]). However, true progress on this issue can only be achieved if a model-independent method is found to determine the chemical decoupling time of individual hadron species.

\section{SPS: final remarks}

The lessons learned from the SPS experiments may be summarized by the following core observations ${ }^{1}$ :

- SPS experiments have created a new state of high energy-density and temperature matter

- sub-hadronic degrees of freedom offer a viable explanation for many of the observed phenomena

- no single observable or measurement is capable of unambiguous proof for the onset of deconfinement

- only a combination of observables and experiments will be able to deliver proof of a new phase of matter

- the concept of a QGP needs to be rethought: in the SPS domain (and perhaps even at RHIC) searching for a fireball of quarks and gluons interacting dominantly according to leading order $\mathrm{pQCD}$ is unrealistic - most likely deconfinement will occur and be observed in the form of a fast evolving phase of partonic degrees of freedom interacting in the domain of soft non-perturbative QCD.

\section{RHIC: first results and expectations}

The Relativistic Heavy Ion Collider has provided an impressive array of data in its first year of operation and QGP theory faces a stiff challenge to keep up with the pace at which new and surprising results emerge. In the following sections a brief overview on some of the most exciting and challenging facets of RHIC theory will be given - a more comprehensive review on the current status can be found in [57].

\footnotetext{
${ }^{1}$ a detailed discussion of charmonium suppression can be found at the end of the RHIC section
} 


\subsection{Initial Conditions}

An issue of major importance for understanding the physics at RHIC is how to describe the initial partonic configuration of the system. In recent years the Color Glass Condensate [58] has emerged as one of the leading concepts in that domain: with decreasing $x$ (the fraction of the longitudinal momentum of the parent hadron), the gluon density in the hadron wavefunction grows faster than the quark density, giving rise to a high-density multiparticle state of gluons. Eventually, the increase in the number of gluons has to cede and saturation, i.e. a limitation of the maximum gluon density per unit phase-space, occurs. Saturation can be characterized by an intrinsic momentum scale $Q_{s}$ (the saturation scale), below which non-linear effects in the parton structure function evolution are expected to slow down and eventually saturate the increase of the gluon density. The saturation scale $Q_{s}$ may also provide a natural cut-off for pQCD based calculations, such as in a parton cascade model. As we shall see in the following sections, the Color Glass Condensate (CGC) approach provides a viable explanation for a number of observations made at RHIC.

\subsection{Hard Processes}

One of the first questions to ask concerning the initial RHIC results is whether evidence for hard processes can be seen and if so, to what extent they dominate particle production and the underlying reaction dynamics: the PHOBOS data on the charged particle multiplicity at midrapidity and its centrality dependence [59] has been analyzed in a variety of different approaches, spanning from saturation model approaches to $\mathrm{pQCD}$ mini-jet production via hard processes [60-62]. Of particular note is a simple analysis carried out in the eikonal approach, decomposing particle production into a hard component, which scales with the number of collisions $\left\langle N_{\text {coll }}\right\rangle$, and a soft component, which scales with the number of participants $\left\langle N_{\text {part }}\right\rangle[60,61]$ :

$$
\frac{d N}{d \eta}=(1-x) n_{p p} \frac{\left\langle N_{\text {part }}\right\rangle}{2}+x n_{p p}\left\langle N_{\text {coll }}\right\rangle
$$

with $n_{p p}$ being the charged particle multiplicity in non-single diffractive $\bar{p} p$ interactions at the respective $\sqrt{s}$. Using parameters extracted from a Glauber calculation and fitting the parameter $x$ to the PHOBOS data this analysis yields a fraction of $37 \%$ of the produced particles stemming from hard processes at $\sqrt{s}=130 \mathrm{GeV}$. The centrality dependence of the charged particle multiplicity is nicely reproduced as well - see the left frame figure 5 (taken from [59]). However, an analogous analysis utilizing the saturation approach of the Color Glass Condensate yields similar agreement with the data, when employing a centrality dependent saturation scale $Q_{s}(b)[60]$ :

$$
\frac{2}{N_{\text {part }}} \frac{d N}{d \eta} \simeq 0.82 \ln \left(\frac{Q_{s}^{2}(b)}{\Lambda_{Q C D}^{2}}\right)
$$

Since both, the conventional QCD and the high-density QCD (i.e. saturation) approach are able the describe the data, it is currently difficult to distinguish between these two approaches. Comparisons to other data, e.g. transverse momentum spectra, will be able to resolve this ambiguity. 

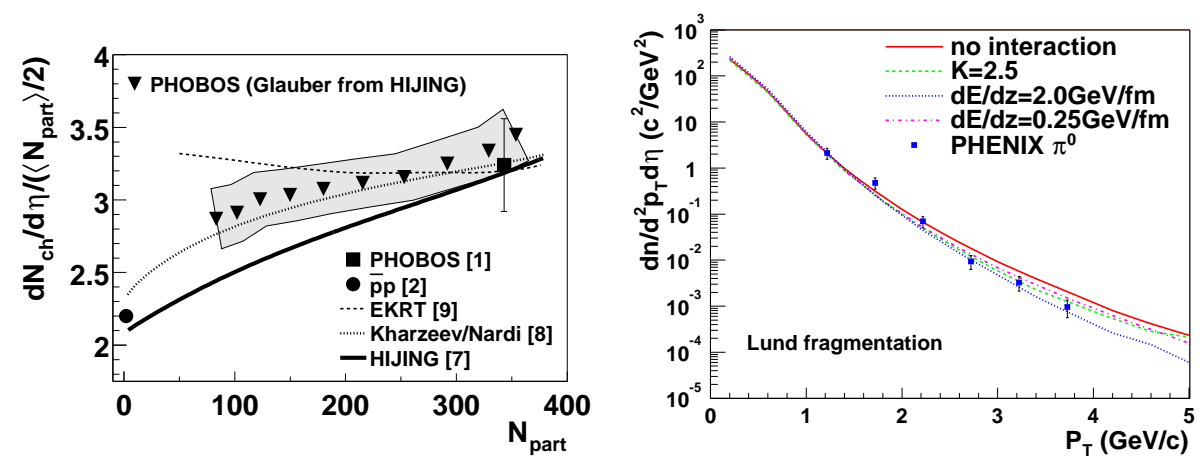

Figure 5. Left: Charged particle multiplicity per participant as a function of centrality measured by the PHOBOS experiment compared to various theoretical calculations. Right: $\pi^{0}$ transverse momentum spectrum measured by PHENIX compared to pQCD based calculations employing different scenarios of partonic energy loss.

Partons created in a hard scattering can act as very efficient probes of a QGP: a parton traversing a color confined medium of hadrons sees a relatively transparent system. However, a parton passing through a hot medium of deconfined degrees of freedom is expected to loose a large amount of energy via gluon radiation. Since the radiated gluons may couple to each other, the energy loss is predicted to be proportional to the square of the length traversed in the deconfined medium [63] - this phenomenon is commonly being referred to as jet-quenching. RHIC experiments have established a significant suppression of high$p_{T}$ hadrons produced in central $\mathrm{A}+\mathrm{A}$ collisions compared to those produced in peripheral $\mathrm{A}+\mathrm{A}$ or binary scaled $\mathrm{p}+\mathrm{p}$ reactions, indicating a strong nuclear medium effect, compatible with the jet-quenching predictions [64,65]. Early calculations predicting a fairly large rate of energy loss around $2 \mathrm{GeV} / \mathrm{fm}$ have been shown to be incompatible with the measured spectra at RHIC $[64,65]$, whereas newer calculations based on the GLV formalism [66] for "thin" plasmas and including finite kinematic effects provide a far better agreement with the data [67] - similar to the assumption of an rather small energy loss around $0.25 \mathrm{GeV} / \mathrm{fm}$.

However, alternative scenarios for partonic energy loss have to be considered: the parton may change its momentum through hard scattering in the deconfined medium [68] or after hadronizing through soft hadronic rescattering [69]. Figure 5 (taken from [68]) shows a parton cascade calculation which compares standard parton energy loss calculations with a collisional energy loss scenario - both approaches are compatible with the PHENIX data [64].

\subsection{Balance Functions and Fluctuations}

While there is no doubt about the importance of partonic degrees of freedom for the initial, early, reaction stages, one of the crucial questions is how long the deconfined state may have actually existed and whether this time-span is long enough for thermalization and collective effects to occur. Balance functions offer a unique model-independent formalism to probe the time-scales of a deconfined phase and subsequent hadronization [70]. If a long-lived QGP has been formed, a large number of quark-antiquark pairs need to be 

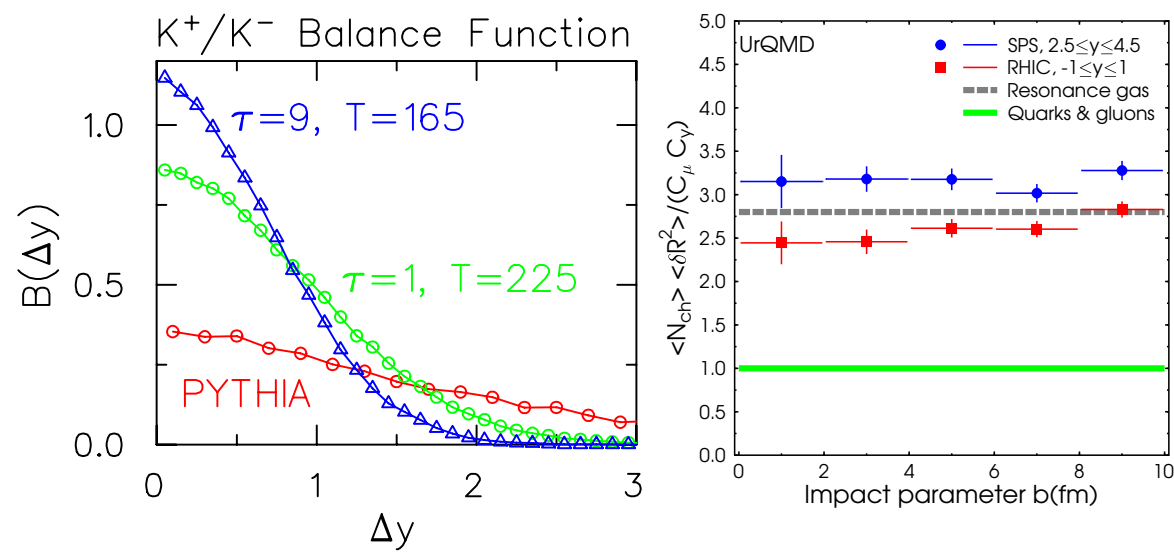

Figure 6. Left: $K^{+} / K^{-}$balance functions calculated for different hadronization times and scenarios. Right: Transport model calculation of charged particle fluctuations compared to analytical predictions for a resonance gas and a QGP.

created close to the hadronization time, mainly due to entropy conservation constraints. This late-stage production of quarks could be attributed to three mechanisms: formation of hadrons from gluons, conversion of the non-perturbative vacuum energy into particles, or hadronization of a quark gas at constant temperature. Hadronization of a quark gas should approximately conserve the net number of particles due to the constraint of entropy conservation. Since hadrons are formed of two or more quarks, creation of quark-antiquark pairs should accompany hadronization. All three mechanisms for late-stage quark production involve a change in the degrees of freedom. Therefore, any signal that pinpoints the time where quarks first appear in a collision would provide valuable insight into understanding whether a novel state of matter has been formed and persisted for a substantial time.

The link between balance functions and the time at which quarks are created has a simple physical explanation. Charge-anticharge pairs are created at the same location in spacetime, and are correlated in rapidity due to the strong collective expansion inherent to a relativistic heavy ion collision. Pairs created earlier can separate further in rapidity due to the higher initial temperature and due to the diffusive interactions with other particles. The balance function, which describes the momentum of the accompanying antiparticle, quantifies this correlation. The balance function describes the conditional probability that a particle in the bin $p_{1}$ will be accompanied by a particle of opposite charge in the bin $p_{2}$ :

$$
B\left(p_{2} \mid p_{1}\right) \equiv \frac{1}{2}\left\{\rho\left(b, p_{2} \mid a, p_{1}\right)-\rho\left(b, p_{2} \mid b, p_{1}\right)+\rho\left(a, p_{2} \mid b, p_{1}\right)-\rho\left(a, p_{2} \mid a, p_{1}\right)\right\},
$$

where $\rho\left(b, p_{2} \mid a, p_{1}\right)$ is the conditional probability of observing a particle of type $b$ in bin $p_{2}$ given the existence of a particle of type $a$ in bin $p_{1}$. The label $a$ might refer to all negative kaons with $b$ referring to all positive kaons, or $a$ might refer to all hadrons with a strange quark while $b$ refers to all hadrons with an antistrange quark.

The left frame of figure 6 shows $K^{+} / K^{-}$balance functions as predicted in a simple

Pramana - J. Phys. 
Bjorken thermal model for two hadronization temperatures, $225 \mathrm{MeV}$ and $165 \mathrm{MeV}$ as well as for fragmenting strings (utilizing PYTHIA), which would represent the hadronization scenario of a hadronic/string picture similar to that of RQMD or UrQMD. Since particles from cooler systems have smaller thermal velocities, they are more strongly correlated in rapidity and result in narrower balance functions. A strong sensitivity to the hadronization temperature and time can be clearly observed. The STAR collaboration has recently published first results on a charged particle balance function analysis at RHIC [71]: a striking centrality dependence is observed, with the width of the balance function decreasing as a function of increasing centrality, in line with what to expect in the case of a long-lived deconfined phase.

The size of the average fluctuations of net baryon number and electric charge in a finite volume of hadronic matter differs widely between the confined and deconfined phases, due to the different value of the elementary charge carried by quarks vs. hadrons. These differences may be exploited as indicators of the formation of a quark-gluon plasma in relativistic heavy-ion collisions, if the fluctuations created in the initial state survive until freeze-out [73,74]. The right frame of figure 6 (taken from [75]) compares transport calculations in the framework of a string/hadron model for SPS and RHIC with analytical predictions for charge fluctuations in a hadronic resonance gas and a parton gas [75]. Since the transport model does not contain any deconfined degrees of freedom, the numerical calculation agrees well with the resonance gas prediction.

Interestingly, sofar all experimental analysis for SPS and RHIC agree with the hadron gas prediction, giving no indication at all about a possible deconfined phase. Since many other observables are compatible with the assumption of deconfinement, it may very well be that the dynamics of hadronization strongly affects the charge fluctuation observables and masks the deconfined phase.

Under certain assumptions, charge fluctuations can be directly expressed in terms of balance functions [72]:

$$
\frac{\left\langle(Q-\langle Q\rangle)^{2}\right\rangle}{\left\langle N_{\mathrm{ch}}\right\rangle}=1-\int_{0}^{Y} d \Delta y B(\Delta y \mid Y)+O\left(\frac{\langle Q\rangle}{\left\langle N_{\mathrm{ch}}\right\rangle}\right)
$$

The size of the correction term is usually less than $5 \%$ for electrical charge fluctuations, since the number of produced charges is much larger than the net charge. However, for baryon number fluctuations the additional term is not negligible, even at RHIC.

Comparing the apparent lack of QGP indications of the charge fluctuation observables with the promising results of the balance function analysis one is lead to speculate whether relevant dynamical information of the two particle correlation is averaged out by integrating over the correlation function in order to obtain the fluctuation observable.

\subsection{Collective Flow}

The study of deconfinement and a subsequent phase-transition to deconfined hadronic matter poses a great challenge to microscopic transport models. In most approaches hadronization is a uni-directional process and the equation of state of the system ill-defined. One possible remedy is to use a hydrodynamical approach for the early deconfined phase of the reaction and subsequent phase-transition, coupled with a microscopic calculation for the later, hadronic, reaction phase in which the hydrodynamical assumptions are not valid any 

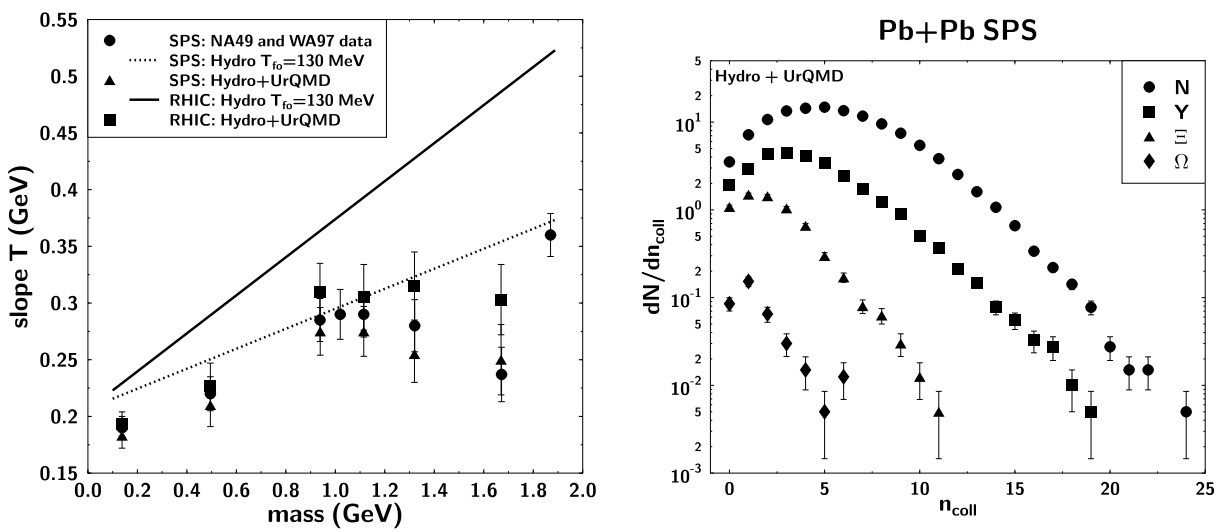

Figure 7. Left: Inverse hadron $m_{T}$ slopes at $y_{c . m} .=0$. The lines depict pure hydrodynamics whereas the symbols refer to data and hydro+micro calculations. Right: collision number distributions for different baryon species in the hadronic phase of a hydro+micro calculation.

longer [21]. In the following, such a combined macro+micro model will be used to study elliptic flow as well as the flavor- and mass-dependence of hadronic slope parameters (ie. radial flow):

Radial and elliptic flow in non-central heavy ion collisions can constrain the effective Equation of State(EoS) of the excited nuclear matter. It has been shown that for an EoS with a first order phase transition, the above mentioned hybrid macro+micro models reproduce both the radial and elliptic flow data at the SPS $[21,76]$. The centrality dependence of the elliptic flow coefficient $v_{2}$ exhibits a strong sensitivity to the underlying EoS, which may help to constrain the EoS [77]. In addition, a number of features of the RHIC data can also be explained within the hybrid approach [77]: the observed elliptic flow and its dependence on $p_{T}$ and mass, the anomalous $\bar{p} / \pi^{-}$ratio for $p_{T} \approx 2.0 \mathrm{GeV}$, and the difference in the impact parameter dependence of the $\bar{p}$ and $\phi$ slope parameters. For an EoS without the hard and soft features of the QCD phase transition, the broad consistency with the data is lost.

The left frame of figure 7 displays the inverse slope parameters $T$ obtained by an exponential fit to $d N_{i} / d^{2} m_{T} d y$ in the range $m_{T}-m_{i}<1 \mathrm{GeV}$ for SPS and RHIC in a hybrid hydro+micro calculation and compares them to SPS data [31]. The trend of the data, namely the "softer" spectra of $\Xi$ 's and $\Omega$ 's as compared to a linear $T(m)$ relation is reproduced reasonably well. This is in contrast to "pure" hydrodynamics with kinetic freeze-out on a common hypersurface (e.g. the $T=130 \mathrm{MeV}$ isotherm), where the stiffness of the spectra increases linearly with mass as denoted by the lines in fig. 7 . When going from SPS to RHIC energy, the model discussed here generally yields only a slight increase of the inverse slopes, although the specific entropy is larger by a factor of 4-5 ! The reason for this behavior is the first-order phase transition that softens the transverse expansion considerably.

The softening of the spectra is caused by the hadron gas emerging from the hadronization of the QGP being almost "transparent" for the multiple strange baryons. This can be seen by calculating the average number of collisions different hadron species suffer in

Pramana - J. Phys. 

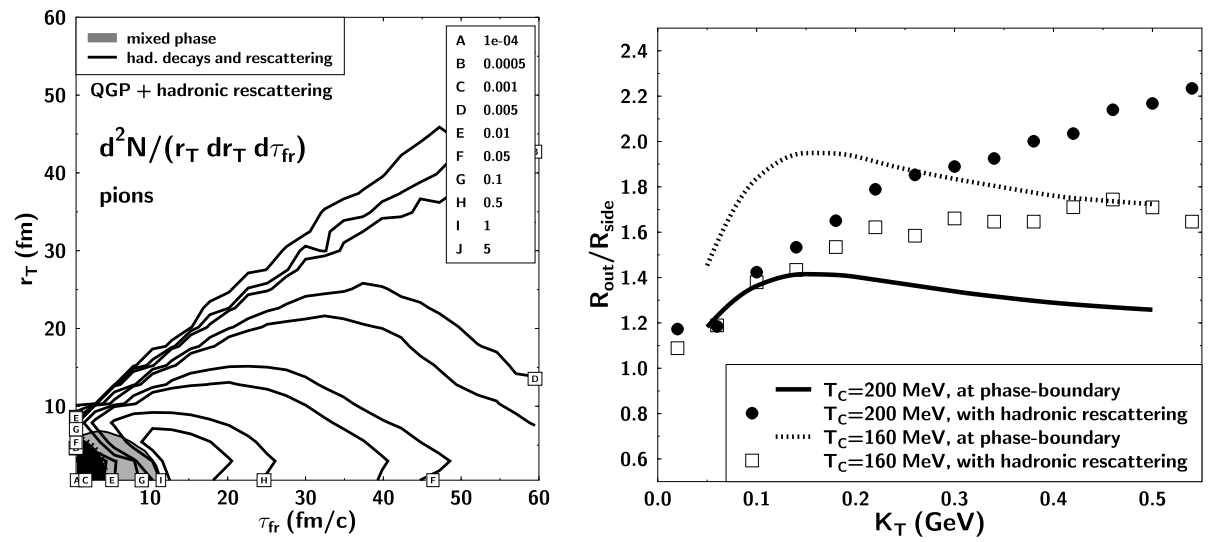

Figure 8. Left: Freeze-out transverse radius and time distribution $d^{2} N /\left(r_{T} d r_{T} d t_{f r}\right)$ for pions at RHIC. Right: $R_{\text {out }} / R_{\text {side }}$ for RHIC initial conditions, as a function of $K_{T}$ at freeze-out (symbols) and at hadronization (lines).

the hadronic phase (see right frame of fig. 7): whereas $\Omega$ 's suffer on average only one hadronic interaction, $N$ 's and $\Lambda$ 's suffer approx. 5-6 collisions with other hadrons before they freeze-out.

Thus, one may conclude that the spectra of $\Xi$ 's and especially $\Omega$ 's are practically unaffected by the hadronic reaction stage and closely resemble those on the phase boundary. They therefore act as probes of the QGP expansion prior to hadronization and can be used to measure the expansion rate of the deconfined phase.

\subsection{Two Particle Interferometry}

Kinetic freeze-out, at which all (elastic) interactions cease, terminates the evolution of the reaction discussed in figure 2. However, the freeze-out time of the system is ill-defined, since interactions cease locally on a particle per particle basis. The left frame of fig. 8 shows the distribution of freeze-out points of pions in the forward light-cone, as well as the hadronization hypersurface from where all hadrons emerge in a hydro+micro approach. Freeze-out is seen to occur in a four-dimensional region within the forward light-cone rather than on a three-dimensional "hypersurface" in space-time. Similar results have also been obtained within other microscopic transport models [78] when the initial state was not a QGP. It is clear that the hadronic system disintegrates slowly (as compared to e.g. the hadronization time), rather than emitting a "flash" of hadrons (predominantly pions) in an instantaneous decay.

A first order phase transition leads to a prolonged hadronization time as compared to a cross-over or ideal hadron gas with no phase transition, and has been related to unusually large Hanbury-Brown-Twiss (HBT) radii [79-81]. The phase of coexisting hadrons and QGP reduces the "explosivity" of the high-density matter before hadronization, extending the emission duration of pions [79-81]. This phenomenon should then depend on the hadronization (critical) temperature $T_{c}$ and the latent heat of the transition. For recent reviews on this topic we refer to $[82,83]$. 

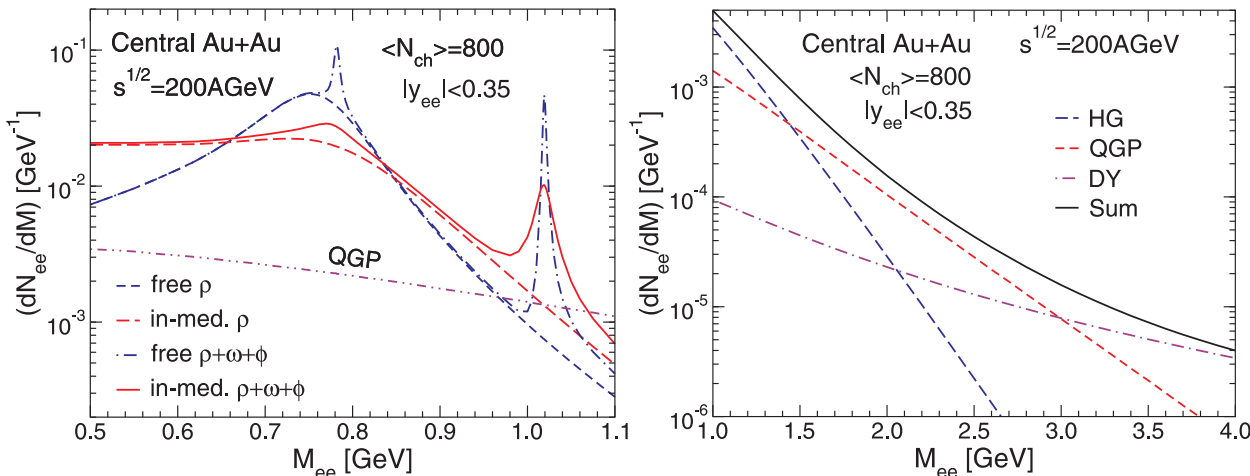

Figure 9. Left: Invariant mass spectrum of low mass dileptons calculated with and without hadronic medium modifications, including possible contributions from thermal dileptons stemming from a QGP. Right: Mass spectrum of intermediate mass dileptons - around $M_{e e} \simeq 2.5 \mathrm{GeV}$ the contribution of QGP radiation dominates above the hadronic background and Drell-Yan processes.

It has been suggested that the ratio $R_{\text {out }} / R_{\text {side }}$ should increase strongly once the initial entropy density $s_{i}$ becomes substantially larger than that of the hadronic gas at $T_{c}$ [81]. The strong $T_{C}$ dependence of $R_{\text {out }} / R_{\text {side }}$ in such a purely hydrodynamical scenario can be seen in the right frame of fig. 8 (solid and dotted lines). Here, the $R_{\text {out }} / R_{\text {side }}$ ratio at hadronization (calculated in a hydrodynamical scenario) is compared to the ratio after subsequent hadronic rescattering and freeze-out in a combined hydro+micro approach [84]. Clearly, up to $K_{T} \sim 200 \mathrm{MeV}$ (with $\left.K_{T}=\left(p_{1, T}+p_{2, T}\right) / 2\right) R_{\text {out }} / R_{\text {side }}$ is independent of $T_{c}$, if hadronic rescatterings are taken into account. Moreover, at higher $K_{T}$ the dependence on $T_{c}$ is even reversed: for high $T_{c}$ the $R_{\text {out }} / R_{\text {side }}$ ratio even exceeds that for low $T_{c}$. A higher $T_{c}$ speeds up hadronization but on the other hand prolongs the dissipative hadronic phase that dominates the HBT radii. This is because during the non-ideal hadronic expansion the scale of spatial homogeneity of the pion density distribution increases, as the pions fly away from the center, but the transverse flow can hardly increase to counteract. Therefore, after hadronic rescattering $R_{\text {out }} / R_{\text {side }}$ does not drop towards higher $K_{T}$ (in the range $K_{T} \lesssim 3 m_{\pi}$ ).

For central collisions of $\mathrm{Au}$ nuclei at $\sqrt{s}=130 \mathrm{~A} \mathrm{GeV}$, data from STAR gives $R_{\text {out }} / R_{\text {side }} \simeq 1.1$ at small $K_{T}$ [85]. With increasing $K_{T}$ the data remain flat or even decrease slightly - a trend which is currently not understood theoretically and cannot be reproduced by any of the dynamical models on the market. Kaon interferometry may provide crucial insight into this so-called HBT-puzzle at RHIC [86]: the kaon phase-space density is much lower than that of the pions, thus dramatically reducing higher order correlation effects. In addition, high- $p_{t}$ kaons are emitted to a larger fraction directly from the phase-boundary, increasing the sensitivity to the QGP equation of state.

\subsection{Dileptons and Charm}

First dilepton and charm measurements will soon be forthcoming at RHIC and are highly anticipated: in the dilepton sector, current theoretical expectations are that in the low mass

Pramana - J. Phys. 
region thermal dileptons from a QGP will be strongly suppressed compared to hadronic contributions - mainly from in-medium pion-pion annihilation. Current state of the art calculations point towards a strong broadening of vector mesons in medium [87] - the $\rho$ and $\omega$ peaks should be almost completely dissolved and the $\phi$ significantly broadened (see left frame of figure 9, taken from [87]). At intermediate invariant masses (around $2-2.5 \mathrm{GeV}$ ) thermal dileptons radiated from a QGP start to outshine hadronic sources $[88,89,87]$, while for higher masses the yield of Drell-Yan processes exceeds that of thermal dileptons from a QGP (see right frame of figure 9). Open charm may give a large contribution to the spectrum in the intermediate mass range as well - however independent measurements of open charm will allow to subtract that contribution from the invariant mass spectrum and thus will allow a measurement of the thermal contribution [90,87].

In the charmonium sector, predictions on what to expect at RHIC vary widely - from total suppression to a strong enhancement of the $J / \psi$ and $\psi^{\prime}$ yield! Originally it was predicted that the suppression of heavy quarkonia-mesons could provide one of the signatures for deconfinement in QCD at high temperatures [91]. The idea was based on an analogy with the well known Mott transition in condensed matter systems. At high densities, Debye screening in a quark-gluon plasma reduces the range of the attractive force between heavy quarks and antiquarks, and above some critical density screening prevents the formation of bound states. The larger bound states are expected to dissolve before the smaller ones as the temperature of the system increases. The $\psi^{\prime}$ and $\chi_{c}$ states are thus expected to become unbound just above $T_{c}$, while the smaller $\psi$ state may only dissolve above $\approx 1.2 T_{c}$. Heavier $b \bar{b}$ states offer the same features as $c \bar{c}$ states, but require much shorter screening lengths to dissolve [92]. The $\Upsilon(b \bar{b})$ state may dissolve only around $2.5 T_{c}$, while the larger excited $\Upsilon^{\prime}$ could also dissolve near $T_{c}$.

At SPS energies, less than one charmonium is on average produced per heavy-ion reaction $[93,94]$ - here the screening argument is most compelling and the debate has shifted towards competing hadronic processes [95]: the size of the hadronic absorption cross sections for charmonia remains a contentious issue. However, these cross sections are necessary to estimate the contribution of the hadronic phase to charmonium suppression and unless a more solid understanding of these cross sections is obtained, the interpretation of the SPS charmonium data will remain under debate. At RHIC, several $\bar{c} c$ pairs may be created in one event - thus allowing for a coalescence of a $\bar{c} c$ pair which was not produced in the same hard process. Estimates taking this effect into account actually predict an enhancement of charmonium production in a QGP, compared to a purely hadronic scenario [96].

\section{Outlook}

Thanks to the vast amount of exciting new RHIC data, QGP theory is currently the most active, dynamic and exciting field in theoretical nuclear physics. Already the current data are of such high quality that they might allow not only the observation of a deconfined phase of matter, but also the characterization of the QGP equation of state! In order to accomplish this feat, a consistent picture of all phases of a heavy-ion reaction needs to be developed, not limited to a few chosen observables. Proper baselines for comparison need to be established as well - the importance of the RHIC $\mathrm{p}+\mathrm{p}$ and $\mathrm{p}+\mathrm{A}$ program cannot be overstated: these experiments will serve to constrain theories and models as well as help 
to generate a better understanding of initial state effects.

There are a number of milestones which QGP theory needs to address in the forthcoming years to fulfill the promise the data is currently showing us:

- the development of reliable lattice gauge calculations at finite temparature and chemical potential

- the determination of hadron properties at high densities and temperatures

- generating a better understanding on the mechanisms of hadronization, both, for elementary hadron-hadron reactions as well as for bulk QCD matter

- the link-up of initial state saturation models to the later-stage dynamics of a heavyion collision, including $\mathrm{pQCD}$ processes

- the investigation of mechanisms for chemical equilibration, both in the confined as well as in the deconfined phase

- the development of a transport theory based on QCD which treats both, hard as well as soft processes consistently.

\section{Acknowledgments}

This work was supported by RIKEN, Brookhaven National Laboratory and DOE grants DE-FG02-96ER40945 and DE-AC02-98CH10886.

\section{References}

[1] J. W. Harris and B. Muller, Ann. Rev. Nucl. Part. Sci. 46, 71 (1996);

S. A. Bass, M. Gyulassy, H. Stöcker and W. Greiner, J. Phys. G25, R1 (1999);

J. L. Nagle, arXiv:nucl-ex/0109016; G. Odyniec, arXiv:nucl-ex/0110012.

[2] J. C. Collins and M. Perry. Phys. Lett. 34 (1975) 1353.

[3] A. M. Polyakov. Phys. Lett. B72 (1977) 224.

[4] K. Wilson. Phys Rev. D10 (1974) 2445.

[5] see, for instance, M. Creutz, Quarks, gluons and lattices (Cambridge University Press, Cambridge 1983).

[6] E. Laermann. Nucl. Phys. A610 (1996) 1.

[7] F. Karsch, arXiv:hep-lat/0106019.

[8] F. R. Brown et al.. Phys. Rev. Lett. 65 (1990) 2491.

[9] C. DeTar. Phys. Rev. D37 (1988) 2328.

[10] Z. Fodor and S. D. Katz, arXiv:hep-lat/0106002.

[11] C. Spieles, H. Stöcker and C. Greiner. Phys. Rev. C57 (1998) 908.

[12] B. Barrois, Nucl. Phys. B129, 390 (1977);

D. Bailin and A. Love, Phys. Rept. 107, 325 (1984);

M. G. Alford, K. Rajagopal and F. Wilczek, Phys. Lett. B 422, 247 (1998);

T. Schafer and F. Wilczek, Phys. Rev. Lett. 82, 3956 (1999);

R. Rapp, T. Schafer, E. V. Shuryak and M. Velkovsky, Annals Phys. 280, 35 (2000);

R. D. Pisarski and D. H. Rischke, Phys. Rev. D 61, 074017 (2000). 
[13] K. Rajagopal and F. Wilczek, At the Frontier of Particle Physics / Handbook of QCD', M. Shifman, ed., (World Scientific), arXiv:hep-ph/0011333.

[14] L. McLerran and R. Venugopalan, Phys. Rev. D 49, 3352 (1994); Phys. Rev. D 49, 2233 (1994).

[15] W. Poschl and B. Muller, Phys. Rev. D 60, 114505 (1999); S. A. Bass, B. Muller and W. Poschl, J. Phys. GG25, L109 (1999).

[16] K. Geiger, Phys. Rept. 258, 237 (1995).

[17] X. Wang and M. Gyulassy, Phys. Rev. D 44, 3501 (1991).

[18] S. A. Bass, M. Hofmann, M. Bleicher, L. Bravina, E. Zabrodin, H. Stoecker and W. Greiner, Phys. Rev. C 60, 021901 (1999).

[19] J.D. Bjorken, Phys. Rev. D27, 140 (1983); K. Kajantie and L. McLerran, Nucl. Phys. B214, 261 (1983).

[20] A. Dumitru and D. Rischke, Phys. Rev. C59, 354 (1999).

[21] S. A. Bass and A. Dumitru, Phys. Rev. C61, 064909 (2000).

[22] D. Teaney, J. Lauret and E. V. Shuryak, nucl-th/0011058.

[23] H. Sorge, H. Stocker and W. Greiner, Annals Phys. 192, 266 (1989).

[24] S. A. Bass et al., Prog. Part. Nucl. Phys. 41, 225 (1998); M. Bleicher et al., J. Phys. G25, 1859 (1999).

[25] see

http: //cern.web.cern.ch/CERN/Announcements/2000/NewStateMatter/

[26] U. Heinz and M. Jacob, nucl-th/0002042.

[27] J. Rafelski, B. Müller, Phys. Rev. Lett. 48, 1066 (1982); (E) 562334 (1986).

[28] P. Koch, B. Müller, J. Rafelski, Phys. Rep. 142, 167 (1986).

[29] P. Koch, B. Müller, H. Stöcker, W. Greiner, Mod. Phys. Lett. A3, 737 (1988).

[30] S. Soff, S. A. Bass, M. Bleicher, L. Bravina, E. Zabrodin, H. Stocker and W. Greiner, Phys. Lett. B471, 89 (1999).

[31] E. Andersen et al. (WA97 collaboration), Phys. Lett B433 209 (1998). R. Lietava et al. (WA97 collaboration), Journal of Physics G25 181 (1999). R. Caliandro et al. (WA97 collaboration), Journal of Physics G25 171 (1999).

S. Margetis et al. (NA49 collaboration), Journal of Physics G25 189 (1999).

F. Gabler et al. (NA49 collaboration), Journal of Physics G25 199 (1999).

D. Evans et al. (WA85 and WA94 collaborations), Journal of Physics G25 209 (1999).

[32] T. S. Biro, H. B. Nielsen, J. Knoll, Nucl. Phys. B245 (1984) 449;

J. Knoll, Z. Phys. C38 (1988) 187;

H. Sorge, M. Berenguer, H. Stöcker, W. Greiner, Phys. Lett. B289 (1992) 6.

[33] S. E. Vance and M. Gyulassy, Phys. Rev. Lett. 83, 1735 (1999).

[34] R. Rapp and E. V. Shuryak, Phys. Rev. Lett. 86, 2980 (2001).

[35] C. Greiner and S. Leupold, J. Phys. G 27, L95 (2001).

[36] J. Kapusta, P. Lichard and D. Seibert. Phys. Rev. D44 (1991) 2774. [Erratum: ibid D47 (1993) 4171.]

[37] E. V. Shuryak. Sov. J. Nucl. Phys. 28 (1978) 408.

[38] B. Sinha. Phys. Lett. B128 (1983) 91.

[39] R. C. Hwa and K. Kajantie. Phys. Rev. D28 (1985) 1109.

[40] C. Song. Phys. Rev C47 (1993) 2861.

[41] L. Xiong, E. V. Shuryak and G. E. Brown. Phys. Rev. D46 (1992) 3798.

[42] J. Alam, D. K. Srivastava, B. Sinha and D. N. Basu. Phys. Rev. D48 (1993) 1117.

[43] A Dumitru, U. Katscher, J. A. Maruhn, H. Stöcker, W. Greiner and D. H. Rischke. Phys. Rev. C51 (1995) 2166.

[44] J. J. Neumann, D. Seibert and G. Fai. Phys. Rev. C51 (1995) 1460.

[45] D. K. Srivastava, B. Sinha, I. Kvasnikova and C. Gale, Nucl. Phys. A 698, 432 (2002).

[46] W. Cassing, W. Ehehalt and C. M. Ko, Phys. Lett. B363 (1995) 35; 
G. Q. Li, C. M. Ko and G. E. Brown, Phys. Rev. Lett 75 (1995) 4007;

V. Koch and C. Song, Phys. Rev. C 54, 1903 (1996); L. A. Winckelmann et al., Nucl Phys. A610 (1996) 116c;

R. Rapp, G. Chanfray and J. Wambach, Phys. Rev. Lett. 76, 368 (1996);

W. Cassing, E. L. Bratkovskaya, R. Rapp and J. Wambach, Phys. Rev. C 57, 916 (1998);

R. Rapp and J. Wambach, Adv. Nucl. Phys. 25, 1 (2000).

[47] J. Letessier, A. Tounsi and J. Rafelski. Phys. Lett. B292 (1992) 417;

J. Letessier, J. Rafelski and A. Tounsi. Phys. Lett. B321 (1994) 394;

J. Sollfrank, M. Gadzicki, U. Heinz and J. Rafelski. Z. Phys. C61 (1994) 659;

J. Letessier, A. Tounsi, U. Heinz, J. Sollfrank and J. Rafelski. Phys. Rev. D51 (1995) 3408;

P. Braun-Munzinger, J. Stachel, J. P. Wessels and N. Xu. Phys. Lett. B365 (1996) 1

F. Becattini, J. Cleymans, A. Keranen, E. Suhonen and K. Redlich, hep-ph/0002267.

[48] P. Braun-Munzinger, I. Heppe and J. Stachel, Phys. Lett. B 465, 15 (1999).

[49] P. Braun-Munzinger, Nucl. Phys. A663-664 (2000) 183.

[50] J. Kapusta and A. Mekjian. Phys. Rev. D33 (1986) 1304.

[51] T. Matsui, B. Svetisky and L. D. McLerran. Phys. Rev. D34 (1986) 2047.

[52] D.M. Elliott and D.H. Rischke. Nucl. Phys. A671 (2000) 583.

[53] T. Biro, E. van Doorn, B. Müller, M. H. Thoma and X. N. Wang. Phys. Rev. C48 (1993) 1275.

[54] K. Geiger and J. I. Kapusta. Phys. Rev. D47 (1993) 4905.

[55] S. A. Bass, P. Danielewicz, S. Pratt and A. Dumitru, J. Phys. G 27, 635 (2001).

[56] L. V. Bravina et al., Phys. Rev. C 63, 064902 (2001) [arXiv:hep-ph/0010172].

[57] L. D. McLerran, hep-ph/0202025, to appear in Pramana.

[58] L. D. McLerran and R. Venugopalan, Phys. Rev. D 49, 2233 (1994);

L. D. McLerran and R. Venugopalan, Phys. Rev. D 49, 3352 (1994);

L. D. McLerran, arXiv:hep-ph/0104285.

[59] B. B. Back et al. [PHOBOS Collaboration], Phys. Rev. C Rapid Comm. in print, arXiv:nuclex/0105011.

[60] D. Kharzeev and M. Nardi, Phys. Lett. B 507, 121 (2001).

[61] X. N. Wang and M. Gyulassy, Phys. Rev. Lett. 86, 3496 (2001).

[62] S. Jeon and J. Kapusta, Phys. Rev. C 63, 011901 (2001).

[63] M. Gyulassy and M. Plumer, Phys. Lett. B 243, 432 (1990); X. N. Wang and M. Gyulassy, Phys. Rev. Lett. 68, 1480 (1992);

R. Baier, Y. L. Dokshitzer, A. H. Mueller, S. Peigne and D. Schiff, Nucl. Phys. B 483, 291 (1997).

[64] K. Adcox et al. [PHENIX Collaboration], Phys. Rev. Lett. 88, 022301 (2002).

[65] C. Adler et al. [STAR Collaboration], Phys. Rev. Lett. 87, 112303 (2001).

[66] M. Gyulassy, P. Levai and I. Vitev, Phys. Rev. Lett. 85, 5535 (2000).

[67] P. Levai, G. Papp, G. Fai, M. Gyulassy, G. G. Barnafoldi, I. Vitev and Y. Zhang, Nucl. Phys. A 698, 631 (2002).

[68] Y. Nara, S. E. Vance and P. Csizmadia, arXiv:nucl-th/0109018.

[69] S. A. Bass et al., Nucl. Phys. A 661, 205 (1999).

[70] S. A. Bass, P. Danielewicz and S. Pratt, Phys. Rev. Lett. 85, 2689 (2000).

[71] G. Westfall and M. Belt-Tonjes for the STAR Collaboration, 18th Winter Workshop on Nuclear Dynamics, January 2002, Nassau, Bahamas, to appear in Heavy Ion Physics.

[72] S. y. Jeon and S. Pratt, arXiv:hep-ph/0110043.

[73] M. Asakawa, U. W. Heinz and B. Muller, Phys. Rev. Lett. 85, 2072 (2000) [arXiv:hep$\mathrm{ph} / 0003169]$.

[74] S. Jeon and V. Koch, Phys. Rev. Lett. 85, 2076 (2000).

[75] M. Bleicher, S. Jeon and V. Koch, Phys. Rev. C 62, 061902 (2000).

[76] D. Teaney, J. Lauret and E. V. Shuryak, Phys. Rev. Lett. 86, 4783 (2001).

Pramana - J. Phys. 
[77] D. Teaney, J. Lauret and E. V. Shuryak, arXiv:nucl-th/0110037.

[78] L. V. Bravina, I. N. Mishustin, N. S. Amelin, J. P. Bondorf and L. P. Csernai, Phys. Lett. B354, 196 (1995); H. Sorge, Phys. Lett. B373, 16 (1996); S. Pratt and J. Murray, Phys. Rev. C 57, 1907 (1998).

[79] S. Pratt, Phys. Rev. D33, 1314 (1986);

[80] B. R. Schlei, U. Ornik, M. Plümer and R. M. Weiner, Phys. Lett. B293, 275 (1992); J. Bolz, U. Ornik, M. Plümer, B. R. Schlei and R. M. Weiner, Phys. Rev. D47, 3860 (1993).

[81] D. Rischke, M. Gyulassy, Nucl. Phys. A608, 479 (1996).

[82] R. M. Weiner, Phys. Rept. 327, 249 (2000); T. Csörgő, hep-ph/0001233.

[83] U. Wiedemann and U. Heinz, Phys. Rept. 319, 145 (1999).

[84] S. Soff, S. A. Bass and A. Dumitru, Phys. Rev. Lett. 86, 3981 (2001).

[85] C. Adler et al. [STAR Collaboration], Phys. Rev. Lett. 87, 082301 (2001).

[86] . Soff, S. A. Bass, D. H. Hardtke and S. Y. Panitkin, Phys. Rev. Lett. 88 in print, arXiv:nuclth/0109055.

[87] R. Rapp, Phys. Rev. C 63, 054907 (2001).

[88] P. V. Ruuskanen. Nucl. Phys. A525 (1991) 255c.

[89] P. V. Ruuskanen. Nucl. Phys. A544 (1992) 169c.

[90] S. Gavin, P.L. McGaughey, P.V. Ruuskanen and R. Vogt. Phys. Rev. C54 (1996) 2606.

[91] T. Matsui and H. Satz. Phys. Lett. B178 (1986) 416.

[92] F. Karsch and H. Satz. Z. Phys. C51 (1991) 209.

[93] D. Kharzeev, Nucl. Phys. A610 (1996) 418;

D. Kharzeev, M. Nardi and H. Satz, Phys. Lett. B405 (1997) 14.

[94] J. P. Blaizot and J. Y. Ollitrault, Phys. Rev. Lett. 77 (1996) 1703.

[95] S. Gavin, M. Gyulassy and A. Jackson, Phys. Lett. B207 (1988) 257;

R. Vogt, M. Prakash, P. Koch and T.H. Hanson, Phys. Lett. B207 (1988) 263;

S. Gavin and R. Vogt, Phys. Rev. Lett. 78 (1997) 1006;

C. Spieles, R. Vogt, L. Gerland, S. A. Bass, M. Bleicher, H. Stocker and W. Greiner, Phys. Rev. C 60, 054901 (1999);

J. Geiss, C. Greiner, E. L. Bratkovskaya, W. Cassing and U. Mosel, Phys. Lett. B 447, 31 (1999).

[96] R. L. Thews, M. Schroedter and J. Rafelski, Phys. Rev. C 63, 054905 (2001). 